\title{
Report of Symposium
}

\section{Infection and immunology in the rheumatic diseases*}

This International Symposium, organized by the World Health Organization and the Arthritis and Rheumatism Council, was held in London on March 19-21, 1974.

\section{Animal models}

Some animal models of possible relevance to infection in the rheumatic diseases in Man were reviewed, including the pig polyarthritides caused by Erysipelothrix (Dr. Drew, U.K.), Clostridia (Dr. Olhagen, Sweden), and Mycoplasma (Dr. Decker, U.S.A.). Similarities of histology between these diseases and rheumatoid arthritis, including nodularity, were pointed out; it was noted that the acute inflammatory response lasted up to 20 weeks in the instance of Erysipelothrix infection. Dr. Decker felt large animals were a much neglected area of study, and collaboration with investigators in veterinary medicine could be valuable.

Dr. Lewis (U.S.A.) summarized experience with a canine colony spontaneously developing a lupus-like syndrome. Cell-free filtrates of spleens from animals with the disease given to mice and normal dogs caused serological abnormalities usually seen in systemic lupus erythematosus (SLE), including the development of antibodies to native DNA and antinuclear antibodies. The recipient mice eventually developed lymphoid tumours; each tumour contained murine leukaemia virus which could be detected by several methods, including the identification of specific surface antigens. One mouse developed a plasma cell tumour which was subsequently studied in tissue culture. The plasma cells produced a specific monoclonal antibody to DNA and C-type RNA virus particles.

Fluorescein-conjugated immunoglobulin prepared from the serum of a girl with SLE reacted with autologous lymphocytes showing a circular rim of immunofluorescence, a pattern also observed in $8 / 10$ other patients with SLE. This reaction was blocked by pretreatment with purified C-type virus, and when the test was repeated, using antibody raised in rabbits to a purified virus preparation produced by the plasma cell tumour, a similar immunofluorescent pattern was found.

\section{Human diseases}

Some rheumatic diseases in which an infectious agent has been found and those in which an infectious cause is probable or possible were discussed at length. Rheumatic fever, polyarteritis nodosa, meningococcal disease, and Yersinia arthritis were included in the former group. Rheumatic fever was cited as an example of the crossreaction between antigens found in micro-organisms (streptococci) and host tissues (heart muscle, glial cells, etc.). Both humoral and cell-mediated immune responses have been shown, though their relative importance is not understood (Dr. L. E. Glynn, U.K.; Dr. Kaplan, U.S.A.; Dr. Zabriskie, U.S.A.). Meningococcal arthritis has been found in a proportion of patients with meningococcal meningitis (Dr. Greenwood, Nigeria). The arthritis developed at the stage of disease when antigen-antibody equivalence could be demonstrated.

Polyarteritis nodosa was associated in some $50 \%$ of patients with immune complex deposition involving Hepatitis B-associated antigen (Dr. Phillips, U.S.A.). Gastrointestinal disease might also represent an area where infectious agents can be identified in arthritic disease, the example of an arthropathy associated with Salmonella and Shigella dysentry being given (Dr. Ansell, U.K.).

The occurrence of an inflammatory polyarthritis during the course of infection with the enteric pathogen Yersinia enterocolitica was reported from Scandinavia (Dr. Larsen, Denmark; Dr. Aho, Finland). This arthropathy has yet to be documented in other areas of the world, but it represents an interesting link between a defined organism and joint disease.

Subsequent papers focused on less definite examples of infection associated with joint disease. The Chlamydiae (formerly Bedsoniae) were thought to be the most likely causative agent of nonspecific urethritis and were found in up to $30 \%$ of patients. There seemed, however, to be some conflict over the interpretation of this finding. Thus, Dr. Schacter (U.S.A.) considered that these organisms were the cause of the infection, whereas Dr. TaylorRobinson (U.K.) had reservations about their significance. The cause of Reiter's syndrome must therefore remain speculative.

Discussions about SLE did not involve a detailed appraisal of the currently conflicting evidence for a viral aetiology, but did include a review of immunodeficiency and its relationship to SLE. The failure of lymphocytes from patients with SLE to show toxicity for fibroblasts from patients with the disease suggested tolerance to a possible pathogen; lymphocytes from most normal subjects being capable of producing a toxic reaction against these fibroblasts (Dr. Russell, Canada). While the evidence in Man is inconclusive, animal models have given more solid but still indirect evidence for a viral cause. The view that SLE is a viral infection seemed nevertheless to be widely held.

Rheumatoid arthritis (RA), a major field of study in this conference, was the subject of several papers. The review of the evidence from morphology, culture, and from antibody studies led Dr. Hamerman (U.S.A.) to suggest that there was inadequate evidence to date to support the belief that rheumatoid arthritis is a viral disease. The indirect evidence, including resistance of synovial cells to rubella and Newcastle disease virus, was

* Report prepared by D. Glass, Charing Cross Hospital and Kennedy Institute, London. 
extended by Dr. Denman (U.K.), who described experiments using herpes simplex. This organism might normally be expected to replicate in transforming lymphocytes, but such replication was inhibited by co-cultivation with rheumatoid synovial cells.

Dr. Smith (U.S.A.) reported that injection of synovial cells from RA patients into rabbit joints induced inclusion bodies and other nuclear abnormalities in the rabbits's synovial cells on subsequent tissue culture, as well as continued resistance to rubella virus.

Dr. Marmion (U.K.) urged caution in the interpretation of evidence from indirect viral studies on mammalian cells in culture, such cells being commonly contaminated with viruses. This need for care was further emphasized by Dr. Hamerman, who commented on reports of termination of the resistance to virus replication in rheumatoid synovial cells by hyaluronidase treatment. In spite of the absence of more positive information relating a virus infection to RA, Dr. Fudenberg (U.S.A.) felt that rheumatoid arthritis would be shown to be a viral infection, but in this context the epithet 'slow' might be better reserved for the virologist rather than the virus!

Negative studies on mycoplasma isolations from rheumatoid joints were reviewed by Dr. Taylor-Robinson. Inhibition of migration by mycoplasma membranes may result from adsorbed IgG (Dr. Maini, U.K.). Dr. Natvig (Norway) could not find evidence of mycoplasma antigens in rheumatoid synovium with fluorescent studies, using mycoplasma-specific antisera.

Professor Duthie (U.K.) discussed the role of diphtheroids found in the rheumatoid joint; an adjuvant effect for these organisms was postulated, a direct pathogenic role being thought unlikely. Dr. Denman reported some indirect evidence on peripheral blood lymphocytes from patients with Behçet's disease which proved to be resistant to the herpes simplex virus during phytohaemagglutinininduced transformation.

\section{Host genetic and other factors}

In some of the examples of infection previously discussed, host factors were implicated. Those subjects developing rheumatic fever were reported to have more streptococcal infections than those who did not (Dr. Zabriskie). In addition, only a proportion of patients with meningococcal meningitis developed polyarthritis and this incidence can change with every epidemic (Dr. Greenwood).

Host factors influencing pathogenicity of a particular organism include genetic background, method of entry to the host, and the immune response. As Dr. Fudenberg emphasized in discussing hypogammaglobulinaemia, lymphoma, and diseases with immunological abnormalities, some of the links between genetic mechanisms and rheumatic diseases were readily detected. Recently, further such markers have become evident. Thus, the leucocyte antigen HLA 27 has been found in a high proportion of patients with sacroiliitis, ankylosing spondylitis, and Reiter's disease (Dr. Brewerton, U.K.). Negroes, said to have a low incidence of sacroiliitis complicating juvenile rheumatoid arthritis and Reiter's disease, when compared to a Caucasian population, also have a lower incidence of HLA 27 (Dr. Ziff, U.S.A.). The reported association of Yersinia arthritis with HLA 27 in 43 of a series of 49 patients may prove to be of value since the organism can be studied, thereby allowing a search for differences in immune responses between those subjects who get arthritis with Yersinia infection and those who do not (Dr. Aho). Patients without HLA 27 who develop Yersinia-associated arthritis have a milder disease than those with the antigen.

Explanations for the role of HLA 27 in disease are being sought; Dr. Pearson (U.S.A.), looking for crossreaction between the antigen and possible pathogens, reported negative results from subjects with HLA 27 and sacroiliitis using a lymphocyte transformation system with a variety of bacteria.

Other associations of HLA antigens and disease can be found in rheumatic fever where Dr. Zabriskie reported the tendency for families with rheumatic fever to share from 1-5 HLA antigens, a feature of less than $30 \%$ of control populations.

Other antigens which could be genetic markers are being evaluated. Dr. Blumberg (U.S.A.) suggested that Hepatitis B antigen will prove to be useful in this respect. This opinion was based on epidemiological studies in which a relationship was found between the presence of the antigen in mothers and in children but was not found between husband and wife. This could be interpreted on both genetic and environmental bases (Dr. Zuckerman, U.K.).

Additional immunodeficiency states associated with disease continue to be described. Professor Lachmann (U.K.) gave examples of patients with complement deficiency (C4 and C2) who had SLE, discoid lupus, or Henoch-Schönlein purpura. Also of interest was the report of an SLE-like disease without antinuclear factors. Similarly, Dr. Peters (U.K.) commented on the association of lipodystrophy, mesangio-capillary glomerulonephritis, and complement deficiencies.

The existence of more subtle immunodeficiency states was suggested by data from Dr. Steward (U.K.), who reported low affinity antibodies to DNA in NZB mice and in patients with SLE. Patients and animals with low affinity antibodies had more severe forms of renal involvement. While the circulating antibody found in the sera may represent only a proportion of the antibody formed, such studies are pointers to wider concepts of immunodeficiency now prevalent.

Dr. Phillips, using established viral-human arthritides as models, reviewed and contrasted the differing degrees of importance of a virus's direct pathogenicity and the host immune response as causes of disease. Arthropathy can result from direct infection of the joint without an immediate host immune response; the infection associated with smallpox vaccination may be such an example. Occasionally during viral infections arthritis can present as an early and transient feature without detection of the organism. This type may result from the toxic effects of virus injury elsewhere, e.g. the Ross River disease; with better understanding, an immune mechanism may account for this type of arthropathy. The third form, that of an immune response directed at the virus localized to the synovium, is characteristic of rubella arthropathy which can persist for several years after the initial infection. The fourth type of arthropathy results from an immune response to the virus at sites remote from the joints with subsequent complex deposition, a serum-sickness type of 
reaction. The association of a polyarthritis at an early phase of Hepatitis B infection might be an example of this type of response, though the exclusion of local complex formation is difficult.

However, Dr. Allison (U.K.) pointed out that genetic predisposition to infectious agents can occur at many levels and some factors, like susceptibility to leukaemogenic virus in birds, depend on control of receptors and are independent of the immune response.

Is genetic background relevant to other chronic rheumatic diseases like rheumatoid arthritis and SLE? Other approaches to this problem, including a study of their epidemiology and the use of such methods to examine the links between genetic and environmental factors, were reviewed by Dr. Carter (U.K.), Dr. Bennett (U.S.A.), and Dr. Lee (U.S.A.). Twin studies do not support strong genetic links in rheumatoid arthritis and the hereditability factors' for RA are no more than can be found for pulmonary tuberculosis. To evoke a Mendelian type of inheritance for rheumatoid arthritis would involve the concept of incomplete penetration to an unacceptable degree. Genetic determinants in rheumatoid arthritis and SLE will be more complex than those shown so consistently between HLA 27 and sacroiliitis.

\section{Persistence of organisms and antigens}

In considering the host factors in the expression of disease, demonstration of persistence of antigen is of particular importance. This has been found in some of the models previously discussed; the Erysipelothrix organism persists for up to 2 years in chronic arthritis of the pig, and in Man rubella has been found for up to 4 months after the onset of arthritis. Experiments relating immune response to the persistence of antigens included those reported by Dr. Oldstone (U.S.A.) who showed prolongation of antigens in the circulation in immune mice compared with nonimmune animals. Similarly nonmicrobial antigen, i.e. bovine serum albumin (BSA), may persist with the appropriate host response (Dr. Jasin, U.S.A.). Animals injected with BSA cleared antigen more quickly than those previously treated with antibody; the complexes of BSA and anti-BSA persisted in collagenous tissues after complexes had been formed in the joint.

Persistence of viral tumour antigens, e.g. polyoma and SV40 virus were described by Dr. Legg (U.K.). These viruses may be incorporated into the host cell genome and replicate new surface antigens without the release of the virus.

The various mechanisms involved were discussed by Prof. Cohen (U.K.), who stressed the ability of microorganisms to persist in the presence of antibody lethal in vitro to the organism. Some of the mechanisms suggested as interfering with host defences included:

(1) Coating of the organism with host antigens.

(2) Blocking of immunologically responsive cells with the organism's antigen.

(3) Intracellular localization of antigen in macrophages.

(4) Production of substances by the organism other than antigens which might inhibit the action of antibody.

Phagosome contents will normally be digested by macrophage lysosomes; on occasions, however, myco- bacteria may be found in phagosomes separated within the cell from lysosomes. In appropriate conditions mycobacteria can be recovered without formation of a phagosome and cell walls have been shown on electron microscopy for up to 10 years after initial infection in spite of apparently adequate therapy (Dr. Rees, U.K.).

Dr. A. A. Glynn (U.K.) reviewed the evidence for bacterial products, so-called impedins, which might inhibit host responses. Virulent bacteria may produce impedins which cause a poorer inflammatory response in the first 6 hours of infection compared with less virulent strains. Virulence could be induced in nonvirulent bacteria strains with corticosteroid therapy or by inhibition of complement.

The persistence of bacterial membranes in macrophages and the inhibition of specific enzyme systems by polysaccharides from mycobacterial cell membranes was reported by Dr. Ginsberg (Israel). Other substances capable of inhibiting lysosomal enzymes were polyionic cations and anions, including histones and DNA. Different bacteria varied in behaviour, for example, ${ }^{14} \mathrm{C}$-labelled Staphylococcus albus could be cleared more quickly than streptococci. It was found that in the instance of streptococci, localization of labelled antigen occurred at remote sites such as burns and in tuberculin sensitivity lesions. These observations may be relevant to organism localization in the joint, for example, adjuvant arthritis.

There is considerable evidence for the persistence of microbiological antigens in tissues over some years in a variety of animals, and on occasions recovery of the live organism.

Some of the methods of study of persistent 'hidden' viral antigens were described (Dr. Legg) and included:

(l) Fluorescent antibodies from patient's serum.

(2) DNA/RNA hybridization.

(3) Demonstration of a reverse transcriptase.

(4) Co-cultivation with standard susceptible cells.

(5) Induction of repressed viruses with carcinogens or $x$-rays.

(6) The addition of part of a virus to supposedly infected cells, from which the intact virus might be produced.

\section{Evaluation of immune status}

Some of the studies previously described, particularly those of antibody affinity, were examples of the use of methods with a high degree of precision in detecting the normal range of immune responsiveness. Other parameters required for assessment of immune status can be measured with less certainty. To relate genetic markers like HLA 27 to immune status of rheumatic disease patients will require a better understanding of some of the available methods. This particularly applies to the quantitation of lymphocyte populations, as well as to the measurements of complexes.

Dr. Lockshin (U.S.A.), commenting on abnormalities of lymphocyte function tests, reported a series of results in which data obtained by lymphocyte transformation were not reflected in other tests, in vivo or in vitro, in the same individual. The difficulty in arriving at a firm consensus about the presence or absence of a lymphocyte response of IgG in rheumatoid arthritis patients might also reflect difficulty in understanding these tests. While inhibition by IgG can be found by using migration studies, the correlation of such results in some instances with IgG 
RFs made interpretation difficult (Dr. Sony and Dr. Clot, France; Dr. Brostoff, U.K.). Supernatants from cells of RA patients containing a migration inhibition factor in response to aggregated $\gamma$-globulin lends support to the view that a cell-mediated response to IgG does exist in rheumatoid arthritis (Dr. Goldberg, U.S.A.).

Dr. Winchester (U.S.A.) reported changes in the estimation of numbers of ' $B$ '-lymphocytes when incubation was performed in different sera. Thus, when cells from a patient were incubated in their own sera a higher number of B-cells were estimated than when the same cell population was incubated in calf serum. Moreover, when these same cells were re-incubated in the patient's serum, the estimated number of B-cells again rose towards the initial value. This effect was probably mediated by complexes or antilymphocyte antibodies. Lymphocytes from patients with glandular fever which cannot be shown to be sensitive to the EB virus by transformation or migration inhibition can be shown to be cytotoxic towards EB virus coated cells (Dr. Denman, U.K.).

Other problems in the evaluation of lymphocyte subclasses were apparent in discussion of $\mathrm{K}$-cells, i.e. those lymphocytes capable of mediating cell lysis in the presence of antibody. These cells, which do not contain immunoglobulins on their surface and are accordingly not B-cells, can be defined on morphological grounds and by functional tests (Dr. Roitt, U.K.; Dr. Natvig, Norway). Rheumatoid rosettes, dependent on the utilization of sensitized sheep cells have not been generally categorized to a particular class (Dr. Bach, France). Dr. Natvig presented evidence, both from morphological and functional tests, that the rheumatoid rosette population (cells with Fc receptors) and cells described as $\mathrm{K}$-cells were the same population and were distinct from both B- and T-cells. The opinion that K-cells were a separate population from both B- and T-lymphocytes was also supported by Dr. Fudenberg. Some of the methodological variables in the detection of rheumatoid rosettes were pointed out by Dr. Bach and Dr. Eibl (Austria). These included differences between the red blood cells of different species and the need to exclude macrophages from the cell populations being studied. It seems that better definition of lymphocyte populations and their function might be a prerequisite to a greater understanding of immunological abnormalities induced by, or causing, these diseases.

Differences exist in measurement of immune complexes with rheumatoid factors and $\mathrm{C1}_{\mathrm{a}}$ these methods utilizing precipitation in gels (Dr. Winchester). Rheumatoid factors will detect as few as two or three aggregated IgG molecules together, whereas $\mathrm{Cl}_{\mathrm{a}}$ requires five IgG molecules as a minimum. The limitation in methods must be considered in selecting them for use in particular situations. For example, both methods will detect the large complexes in rheumatoid synovial fluids, but only rheumatoid factors will generally detect the smaller complexes present in rheumatoid sera. Further, as $\mathbf{C l}_{\mathbf{q}}$ will bind nonspecifically to macromolecules like DNA, analysis of results may be difficult. Before accepting the results from the use of $\mathrm{Cl}_{\mathbf{q}}$, specificity studies with, for example, the use of DNAase, would be desirable. The ${ }^{125} \mathrm{IC1}_{\mathrm{q}}$ binding studies reported by Dr. Lambert (Switzerland) on rheumatoid synovial fluids must be viewed with caution since DNA may be present in these effusions.

\section{Suppression or stimulation of the immune response in the rheumatic diseases?}

In NZB mice and in other relevant animal models, both T-cell suppression and hyper-responsive $\mathrm{B}$-cell populations are found. This situation may exist in SLE and presents a therapeutic dilemma not answered adequately by the present methods using immunosuppressive drugs with global effects. Deficiencies of cellular immunity which may now be reversed with transfer factor were reviewed by Dr. Lawrence (U.S.A.); these included a variety of infections in immunodeficient children and neoplasia.

Of relevance to this meeting was the use of transfer factor to correct immunodeficiency in the rheumatic diseases. Dr. Lockshin was not able to distinguish the impaired T-cell function found in active rheumatoid arthritis with that found in other diseases associated with extensive inflammatory activity. This poor definition of immunodeficiency complicates the use of transfer factor in this situation, though some individuals with rheumatoid arthritis seem to have marked defects in parameters of T-cell activity relatively early in the course of their? disease. Clinical trials have started, Dr. Maini and Dr. Scott (U.K.) reporting a study in rheumatoid arthritis using a 6-week double-blind control trial of cross-over design. No change directly related to the administration of transfer factor could be shown. Dr. Natvig reported the uncontrolled use of transfer factor for a period of up to 5 months in three patients with juvenile rheumatoid arthritis and found clinical improvement. Uncertainty about infectious agents in rheumatoid arthritis complicates the use of transfer factor since selection of 'specific' TF is probably desirable. The selection of donors will be made easier using the recently available in vitro tests for transfer factor when knowledge of a specific antigen is available. Transfer factor has been used in other diseases of uncertain aetiology but in which a virus has been implicated on indirect evidence. Dr. Dupont (U.S.A.) reported the use of transfer factor in multiple sclerosis with which paramyoxovirus agents may be involved. This disease presents, in common with rheumatoid arthritis, great difficulty in the evaluation of any new form of treatment. The outcome of this type of therapeutic approach to immunodeficiency in the rheumatic diseases will be of great interest.

\section{Reference}

Dumonde, D. C. (Editor) (1974) In 'Infection and Immunology in the Rheumatic Diseases'. Blackwell, Oxford. (Conference proceedings to be published shortly). 